\title{
IMPORTANCIA DEL COMPONENTE SOCIAL EN EL MANEJO DEL RECURSO HÍDRICO, RÍO EL ENCANO, HUMEDAL RAMSAR LA COCHA (NARIÑO, COLOMBIA)
}

\author{
Sara María Luna Hernánde $z^{\underline{1}}$ \\ Sandra Milena Madroñero Palacios ${ }^{2}$
}

Recibido el 20 de junio de 2014, aprobado el 17 de abril de 2015 y actualizado el 09 de noviembre de 2015

DOI: 10.17151/luaz.2016.42.13

\section{RESUMEN}

El presente artículo tiene como objetivo general analizar la relevancia de la sociedad en el uso y manejo del recurso hídrico en el río El Encano, fuente hídrica de gran importancia por pertenecer a una de las áreas declaradas como humedal Ramsar en Colombia. Para el logro de este objetivo, se desarrolló el diagnóstico de los diferentes usos del agua en el río. En donde metodológicamente se llevaron a cabo inicialmente salidas de reconocimiento tanto de la zona de estudio como de la población relacionada con el recurso, donde se determinó una población total de 80 familias a las que posteriormente se les aplicó un tipo de encuesta semiestructurada. Así mismo, se georreferenciaron a lo largo del río los usos dados al recurso hídrico por parte de la comunidad, entre estos se resalta las descargas de vertimientos y las captaciones. Finalmente, la información obtenida fue procesada en el programa Excel. De los resultados obtenidos se resalta la identificación de la problemática relacionada con el recurso hídrico en que sobresale el inadecuado manejo de aguas residuales, siendo esta la actividad de mayor impacto en la fuente, así mismo, la ganadería y la agricultura hacen un uso importante del recurso lo que ha contribuido a su deterioro. Además, se establecieron variables sociales como educación y ocupación laboral que cumplen un papel fundamental en la gestión del recurso. Después del análisis de la información obtenida con la investigación, se puede concluir que el río ha sufrido modificaciones de las características físicas y químicas por el permanente proceso de intervención antrópica; de igual manera, se establece que los procesos de gestión del recurso hídrico por parte de la comunidad son incipientes, en donde se hace necesario fortalecer la educación ambiental en toda la población presente en la zona.

\section{PALABRAS CLAVE}

Calidad de agua, diagnóstico, gestión, humedal Ramsar, recurso hídrico.

$$
\begin{gathered}
\text { IMPORTANCE OF THE SOCIAL COMPONENT IN WATER } \\
\text { RESOURCES MANAGEMENT, THE ENCANO RIVER, RAMSAR LA } \\
\text { COCHA WETLAND (NARIÑO, COLOMBIA) }
\end{gathered}
$$

\section{ABSTRACT}

This article aims to analyze the relevance of society in the use and management of water sources in El Encanto River, a water source of great importance because it belongs to one of the areas declared as Ramsar wetland in Colombia. To achieve this objective, a diagnosis of the different uses of the water from the river was conducted through recognition fieldtrips, both in the área of study and in the population, related to the water resource where a population of 80 families was determined and 
applied semi-structured interviews. Likewise, the uses given to water resources by the community along the river were georeferenced, and among them, dumping download and deposits are hignlighted. Finally, the information collected was processed in the Excel Program. From the results obtained, the problem related to water resources was highlighted, where the inadequate management of wastewater stand out being the greatest impact activity in the source, as well as stockbreeding and agriculture which makes important use of the resource and has contributed to its deterioration. Additionally, social variables such as education and job were stablished since they play an important role in the management of the resource. After analyzing the information obtained through this research, it is possible to conclude that the river has suffered modifications of its physical and chemical characteristics due to the permanent anthropic intervention. Similarly, it is established that the processes of water management by the community are incipient, and it is necessary to strengthen environmental education for all population present in the area.

KEY WORDS: Water quality, diagnosis, management, Ramsar wetland, water resources.

\section{INTRODUCCIÓN}

La degradación de los recursos acuáticos es motivo de interés para las ciencias del medio ambiente, para proteger los ecosistemas fluviales y analizar sus cambios en el tiempo, investigándolos de manera integral con el fin de desarrollar criterios que permitan estimar el efecto y magnitud de la intervención antrópica (Araya, 2003). Teniendo en cuenta lo anterior, el presente artículo se centra en uno de los ecosistemas de mayor importancia nacional e internacional por ser declarado como humedal Ramsar, denominación que resalta las características especiales de este ecosistema por ser páramo azonal (The Ramsar Convention on Wetlands, 2011); igualmente, por albergar a la segunda laguna más grande del país. No obstante, a pesar de ser un ecosistema estratégico por los servicios ambientales que presta, en la actualidad es de los más vulnerados. Por otra parte, se hace necesario detallar que la zona de estudio es de gran importancia dado que es el principal tributario de la laguna de La Cocha.

\section{Justificación}

Uno de los aspectos más importantes a resaltar de este estudio, es el aporte al conocimiento de la interacción sociedad-naturaleza, representada en los diferentes componentes sociales, económicos y biofísicos de la zona. Asimismo, se convierte en una herramienta fundamental para los procesos de gestión del recurso hídrico, en donde la identificación de las potencialidades y limitantes es de gran importancia para el establecimiento de soluciones que respondan tanto a las necesidades de la población como del ecosistema. Dentro de los beneficiarios de la investigación se destaca a la comunidad, ya que identificaron y reconocieron los procesos favorables o desfavorables que están desarrollando en relación al recurso hídrico; también, la comunidad académica e instituciones relacionadas, debido a que la información proporcionada se convierte en un elemento importante para ampliar 
conocimientos e identificar las áreas que requieren ser fortalecidas en el humedal.

\section{Problema}

Desconocimiento existente frente al manejo uso y conservación del recurso hídrico en el río El Encano, por parte de la comunidad beneficiaria, respecto de los bienes y servicios ambientales que dicho ecosistema provee.

\section{Objetivo general}

Diagnosticar los usos y el manejo del recurso hídrico en el río El Encano.

\section{MATERIALES Y MÉTODO}

La investigación se desarrolló desde un enfoque cualitativo bajo la orientación del método descriptivo-evaluativo, que permitió el logro del objetivo principal de investigación.

El objeto de estudio fue el río El Encano (Figura 1). La población se constituyó en la comunidad ribereña que abarca el tramo del río correspondiente a 5,596 km de longitud, desde la vereda El Socorro, corregimiento El Encano, municipio de Pasto, con coordenadas $\mathrm{N} 1^{\circ} 10,365^{\prime}$ - W77 $08,496^{\prime}$, hasta su desembocadura en la laguna de La

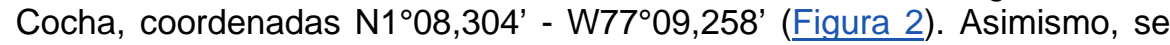
trabajó con la comunidad beneficiada, que se involucra en los procesos de manejo del agua y hace uso de la fuente. Metodológicamente se consideró importante realizar inicialmente una visita de reconocimiento, en donde se identificaron 80 familias que tienen una relación directa con el recurso; debido a que la población de interés era pequeña, se trabajó con la totalidad de la misma. 


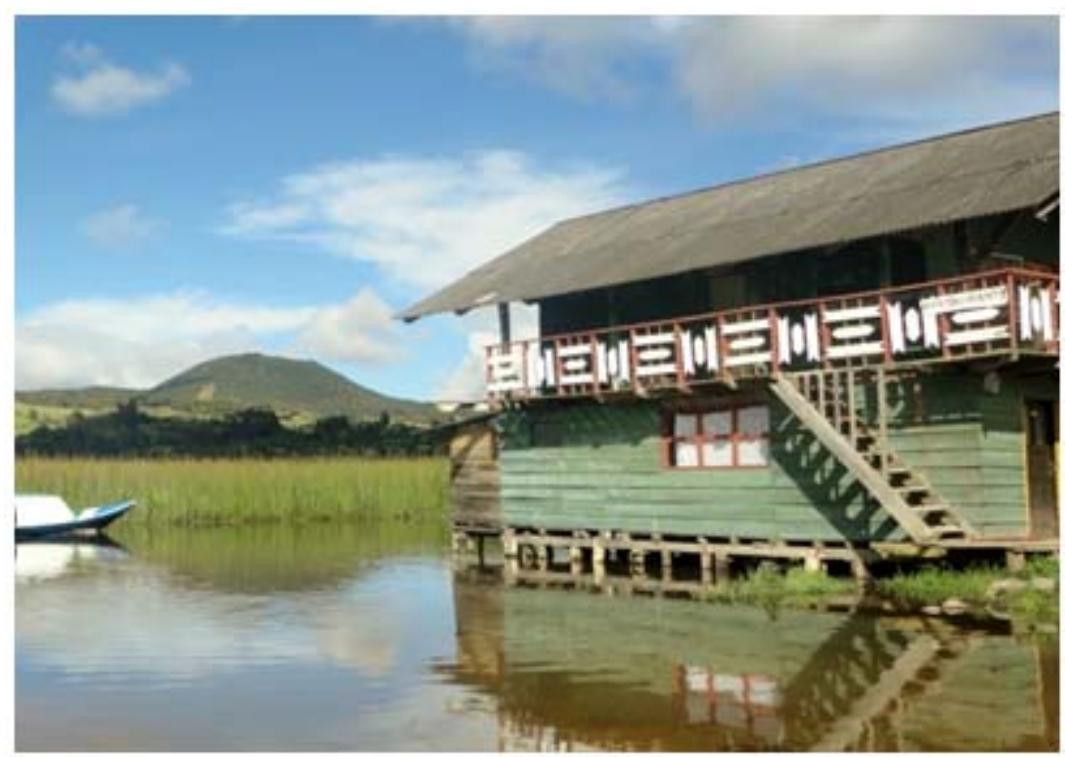

Fuente: Ios autores.

Figura 1. Ubicación de la zona de estudio, río El Encano (Nariño, Colombia).

Posteriormente, se realizó un recorrido a lo largo del río, que permitió identificar los diferentes usos dados por la población, tal es el caso de descarga de vertimientos, descarga de fuentes naturales y las captaciones. Los cuales fueron registrados y georreferenciados.

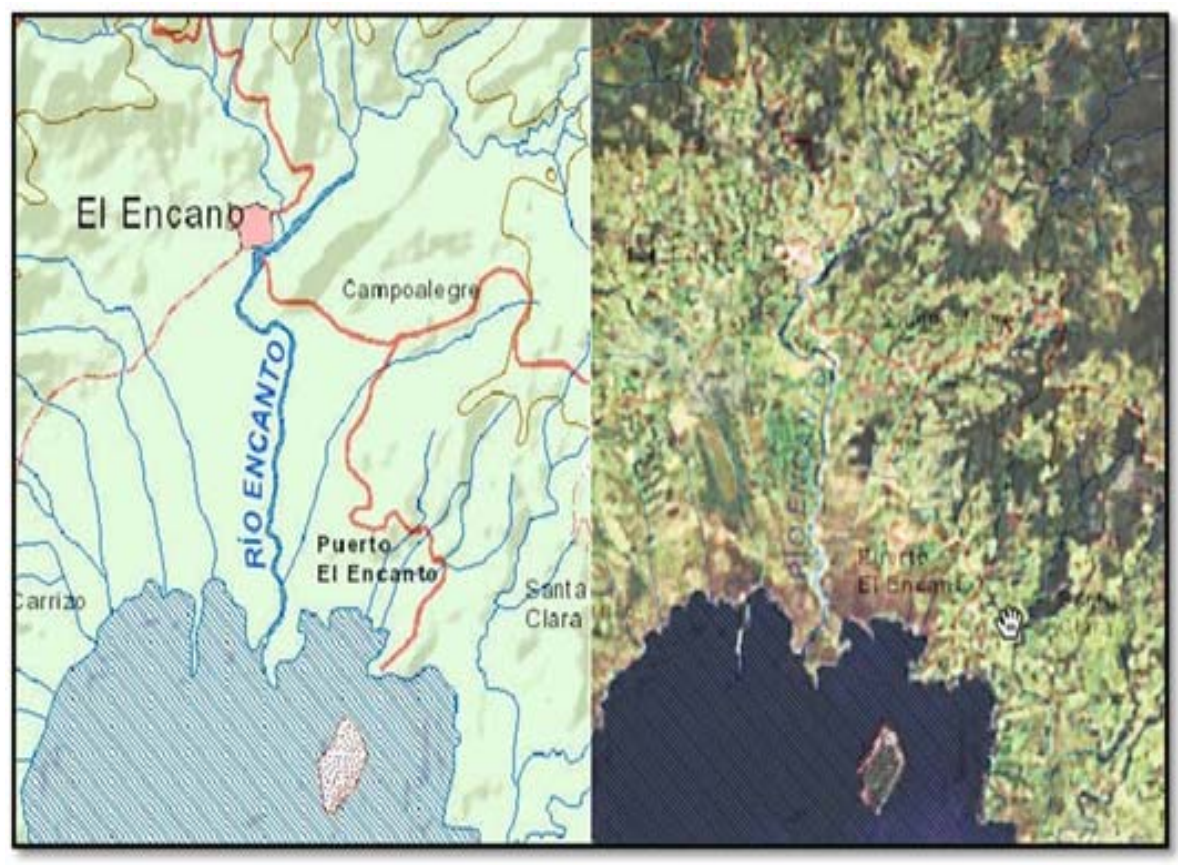

Fuente: IGAC (2014).

Figura 2. Río El Encano, corregimiento de El Encano (Pasto, Nariño). 
Dicha información levantada, se amplió con la aplicación de las encuestas semiestructuradas a las familias identificadas, en donde se realizó una encuesta por cada una de ellas. Esta técnica de diálogo directo con la comunidad evita efectos negativos como temas cerrados, falta de comunicación y adecuación a percepciones de los encuestados (Geilfus, 2009), permitiendo conocer ampliamente la relación de la población con el recurso hídrico; esta técnica, permitió la recolección de información general y específica relacionada con datos básicos del hogar, usos del agua, manejo de aguas residuales, disposición y manejo de residuos sólidos y las problemáticas ambientales dentro del área. Posteriormente, se organizó y sistematizó la información en el programa Microsoft Excel, construyendo la matriz de tabulación de información.

\section{RESULTADOS}

Inicialmente se establece como uno de los principales resultados la identificación de 92 puntos correspondientes a los diferentes usos del recurso (Figura 3), que permite establecer que el uso prioritario y de mayor impacto es la descarga de aguas residuales o vertimientos en la fuente con un porcentaje de 58\%; se debe, fundamentalmente, a que las familias no poseen un sistema de tratamiento de aguas residuales; por lo cual, se descarga directamente al río, esto repercute en las condiciones de calidad, tanto del río El Encano como de la laguna de La Cocha, de la cual es afluente.

Posteriormente, se encuentra la contaminación difusa, originada por actividades provenientes de la agricultura 4\% (Figura 4) y ganadería 11\%; donde la siembra de papa y la presencia de ganado vacuno y especies menores como cerdos, contribuyen a incrementar los procesos de alteración de las condiciones naturales del ecosistema. Se observa la descarga de pequeñas fuentes naturales que aportan su caudal al río, lo que corresponde al $8 \%$, lo cual tiene gran relevancia debido a que contribuye a mejorar los procesos de resiliencia en el río El Encano, dado que entre mayor caudal es mejor la disolución de contaminantes en éste. Finalmente, el $10 \%$ hace referencia a acequias, que son pequeñas zanjas realizadas por los pobladores de la zona para conducir las aguas residuales de fincas y lotes. 


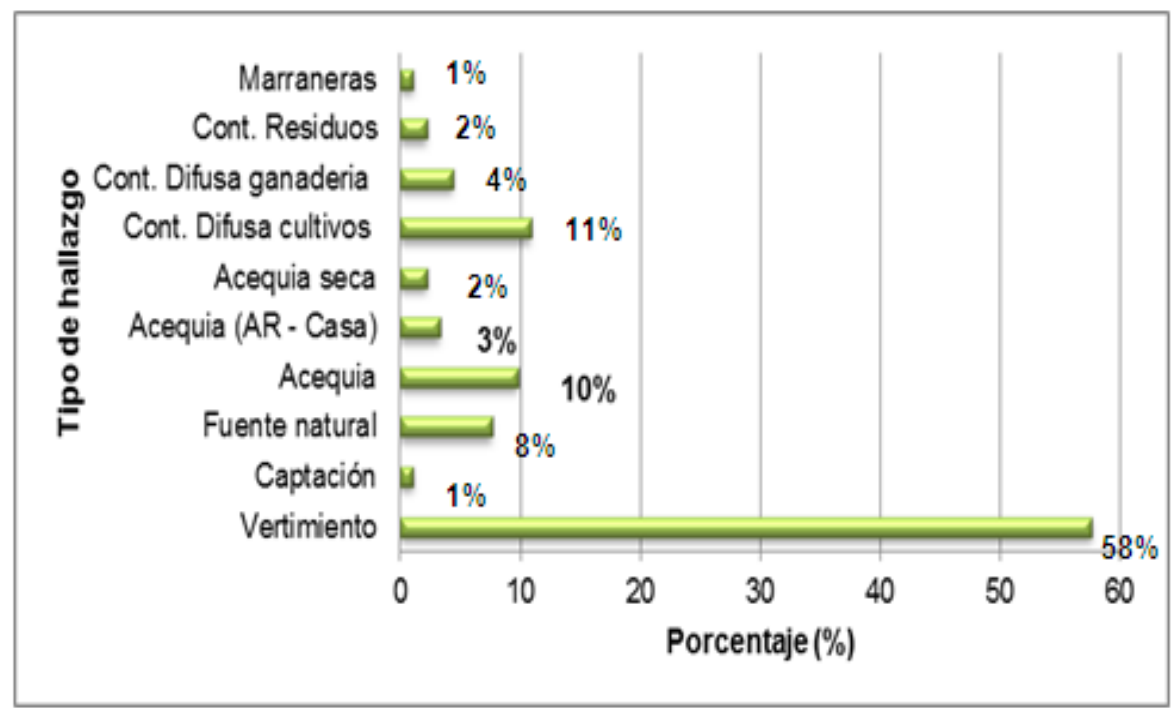

Fuente: los autores.

Figura 3. Hallazgos encontrados en el recorrido de diagnóstico.

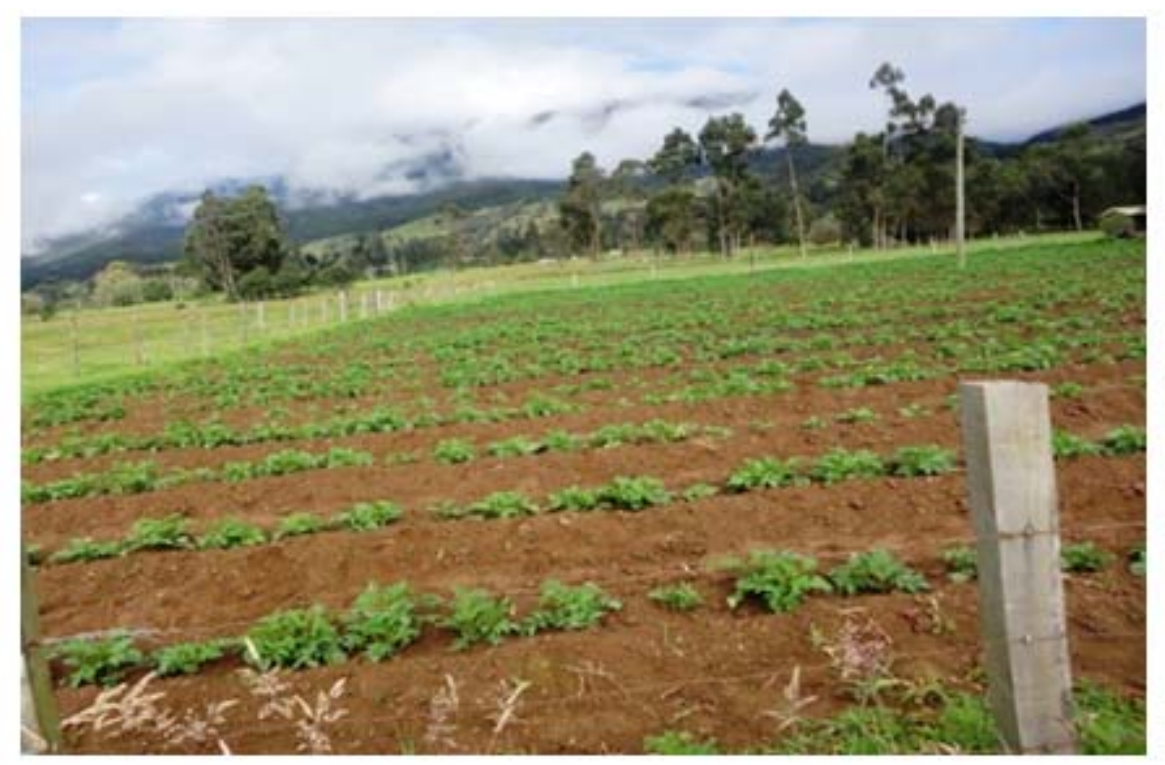

Fuente: Ios autores.

Figura 4. Agricultura en la región, corregimiento El Encano (Nariño).

Por otra parte, se pudieron establecer las diferentes actividades a las cuales se dedica la población de estudio (Figura 5), dentro de las cuales sobresalen la actividad agrícola con un $27 \%$, como una de las de mayor desarrollo, debido a que culturalmente la comunidad ha heredado dicha actividad, sumado al gran potencial ecosistémico que el área ofrece con suelos aptos para el cultivo de diferentes productos; asimismo, se observó que por la dificultad de oferta laboral en la zona muchos de sus habitantes se han visto en la necesidad de buscar trabajo en el área urbana, y los 
que consiguen trabajo dentro de su territorio se emplean como jornaleros para trabajar la tierra (28\%); no obstante, dado que este humedal Ramsar tiene un enorme potencial turístico, el $11 \%$ de la población con cierto nivel de estudios se dedica a esta actividad, transportando a los turistas a lo largo de la laguna en lanchas artesanales.

Finalmente, se hace necesario destacar el papel fundamental que desarrolla la actividad piscícola con un $10 \%$, desde ya hace varios años se ha instaurado el cultivo de trucha como uno de las actividades más importantes para la obtención de recursos económicos; sin embargo, esto ha afectado seriamente la diversidad tanto del río como de la laguna, donde actualmente no se encuentra prácticamente biota nativa en el área, eso sin dejar a un lado, la contribución de dichos cultivos a los procesos de eutrofización del recurso hídrico en la zona.

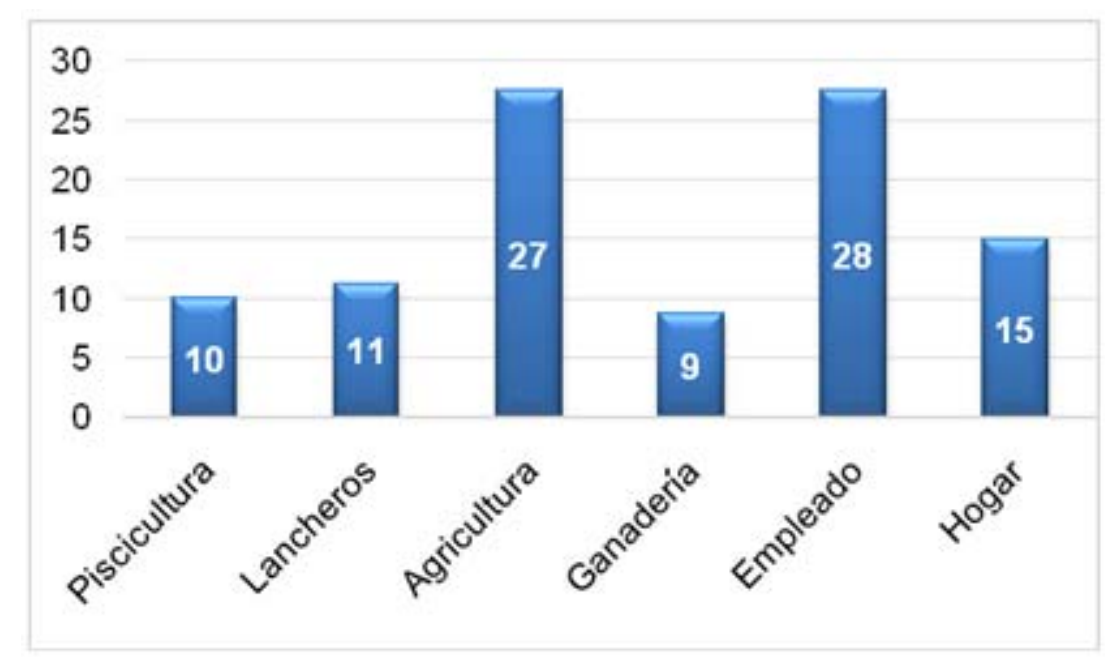

Fuente: los autores.

Figura 5. Principales ocupaciones de los pobladores,

ribera río El Encano.

Respecto a los usos del agua (Figura 6), se consideró importante determinar las actividades en las cuales se usaba mayor cantidad de agua del río El Encano, estableciendo que el $76 \%$ de la población encuestada hace uso del recurso hídrico para la fumigación de cultivos como papa, fresa y hortalizas, entre otros; el 10\% la destina para las actividades de riego, y el $10 \%$ restante hace uso del recurso para lavar los diferentes productos agrícolas sembrados y cosechados en la zona. Es de importancia denotar, que de esta fuente no se extrae agua con fines de consumo humano, ya que sus condiciones de calidad no cumplen con los criterios para dicho uso. 


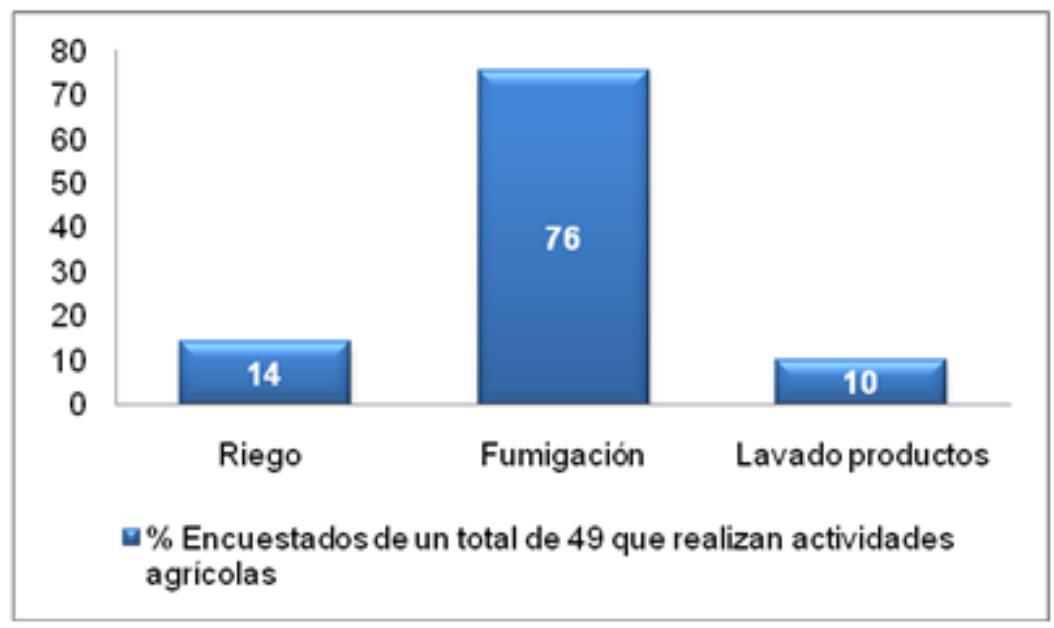

Fuente: Ios autores.

Figura 6. Uso del recurso hídrico en procesos agrícolas.

Otro de los aspectos que fueron considerados dentro del estudio, es la identificación de los principales contaminantes que se encuentran afectando las condiciones fisicoquímicas del río (Figura 7), a lo cual se pudo establecer que el $51 \%$ de la población encuestada descarga las aguas domiciliarias al río, el $31 \%$ deposita los residuos sólidos directamente en el río, el $11 \%$ de la población permite que los animales permanezcan cerca de la fuente, lo que genera contaminación por materia orgánica, y el $6 \%$ restante de la población considera que la contaminación se debe a la actividad agrícola, por la utilización de plaguicidas y pesticidas necesarios para mantener la cantidad de la producción en la zona.

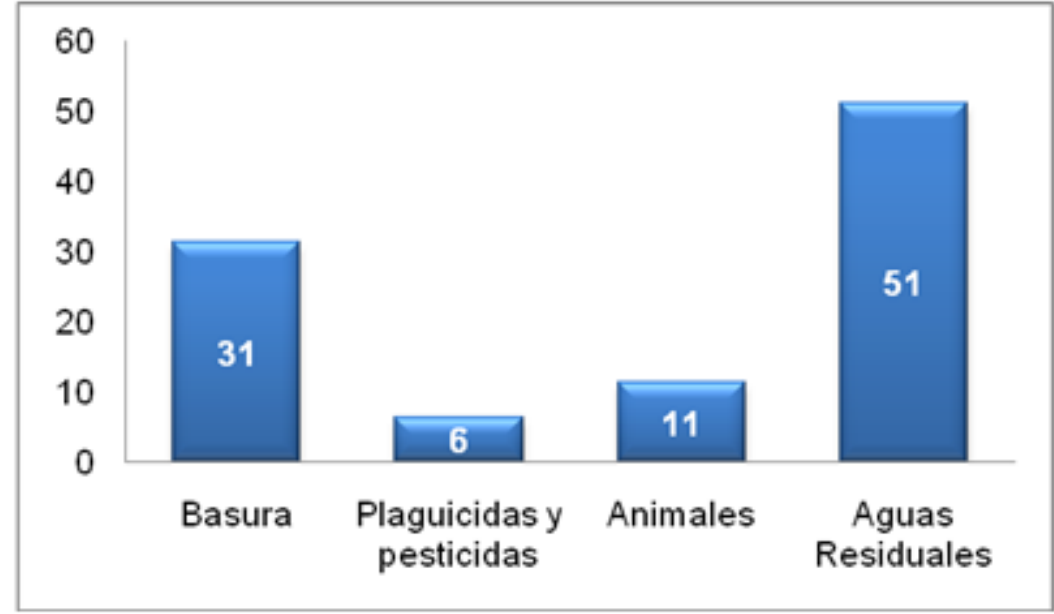

Fuente: Ios autores.

Figura 7. Percepción de la comunidad sobre los principales contaminantes del río El Encano. 
No obstante, es importante resaltar que todas estas actividades generan deterioro del recurso hídrico que afecta tanto a la población como al ecosistema, considerado a este último como uno más de los usuarios. El proceso de deterioro o conservación de los recursos naturales, y especialmente del recurso hídrico, se ve influenciado por la educación ambiental de los usuarios, este aspecto se convierte en uno de los más débiles debido a que el $59 \%$ de la población ha alcanzado únicamente el nivel de básica primaria, el $36 \%$ el de básica secundaria, únicamente el $1 \%$ el nivel universitario y el $4 \%$ no ha recibido ningún tipo de educación (Figura 8); situación que afecta no solo las condiciones de calidad de la población, dado que incrementa la dificultad para la población de competir en el mundo laboral, y el desconocimiento de los aspectos ambientales impide desarrollar prácticas de uso y manejo de los recursos naturales más adecuadas.

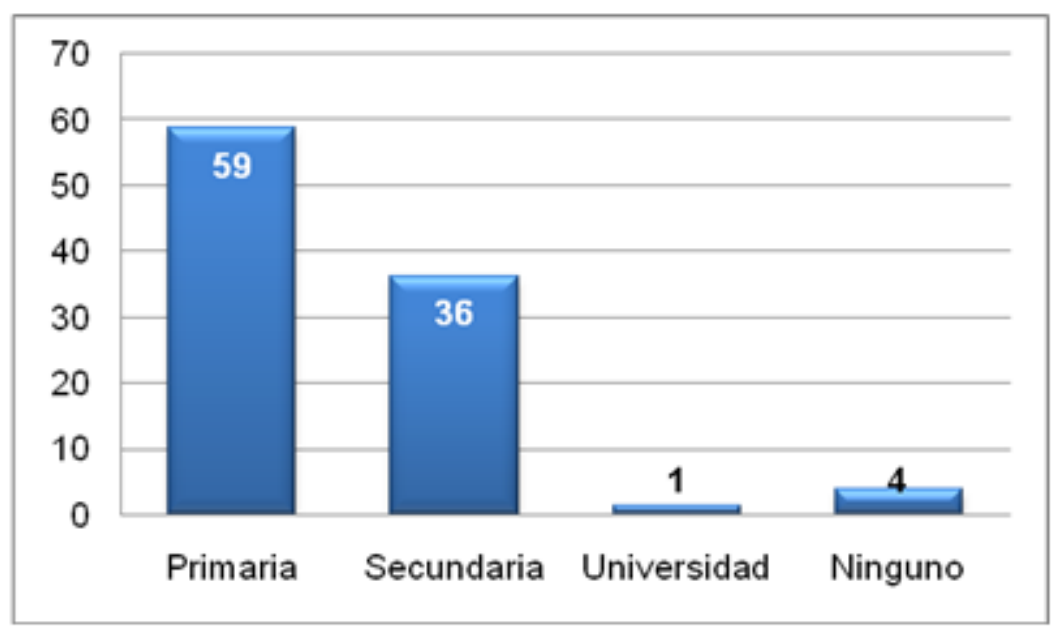

Fuente: Ios autores.

Figura 8. Nivel máximo de escolaridad alcanzado por la población.

Finalmente, la comunidad identificó las opciones más propicias que contribuyen a desarrollar procesos de uso y manejo más sostenibles tanto para la comunidad como para el ecosistema (Figura 9), en donde el $44 \%$ de la población consideró que el ahorro de agua es la actividad más importante para mejorar los procesos de conservación del recurso hídrico, el $20 \%$ propone la necesidad de establecer sistemas de monitoreo que implementen medidores que reduzcan el desperdicio del agua y que obligue a la comunidad a ahorrar el recurso; para el 13\% de la población es importante el control de las fugas de agua, ya que en muchos casos las instalaciones para obtener dicho recurso se construyen artesanalmente; por su parte, el $24 \%$ de la población considera que no es necesario desarrollar ninguna actividad, ya por el momento no presentan ningún inconveniente en cuanto a su salud y disponibilidad del recurso. Esta situación demuestra con claridad la urgencia de promover y fortalecer procesos de educación ambiental en la comunidad, así como también el sentido de pertenencia para contribuir a optimizar y conservar todo el potencial ambiental de este ecosistema. 


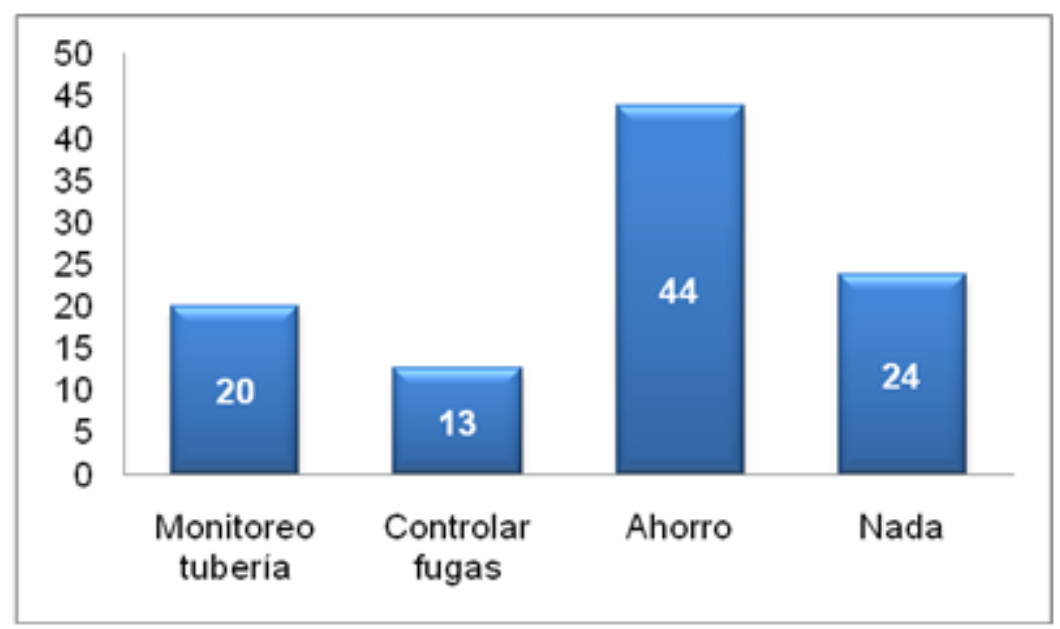

Fuente: Ios autores.

Figura 9. Acciones propuestas para el cuidado del agua.

\section{DISCUSIÓN}

Actualmente es importante analizar la sustentabilidad en el medio rural, ya que en Colombia el $94 \%$ de la población se relaciona con este sector y el $32 \%$ de la misma vive allí (DANE, 2007). Este hecho no es ajeno a la zona objeto de estudio, pues la comunidad encuestada se encuentra ubicada en una zona netamente rural, y es en su gran medida dependiente de los bienes y servicios ambientales generados por el ecosistema. Por lo cual, es fundamental la implementación de procesos de educación ambiental, que contribuyan al mantenimiento de dichos bienes y servicios, ya que, como lo afirma la WWF (2012), el problema del deterioro del recurso hídrico no radica tanto en el crecimiento demográfico, sino en la cultura que la comunidad tiene sobre el agua, cómo la consume y en qué cantidad.

Por su parte, Jaramillo (2006) considera que la presión que se ejerce sobre el recurso hídrico tiene que ver con los cambios en la población rural, pues con el paso de la historia los sectores campesinos han sido referentes básicos para su desarrollo, aunque infortunadamente su inserción en los procesos de construcción social se han presentado sin considerar el desarrollo de sus capacidades como eje central de las políticas y como un sector potencial en sí mismo; en el río El Encano se observa este fenómeno, como en muchos otros sectores del país, en donde existe la necesidad de involucrar activamente a las comunidades en todos los procesos de gestión a implementar dentro de ella, pues su intervención contribuye a mejorar las condiciones de calidad de vida de las poblaciones. Al respecto, Baribbi y Spijkers (2011) aseguran que la mayoría de los hogares rurales viven en condiciones de pobreza o pobreza extrema, $65 \%$ y $33 \%$ respectivamente; además, registran acceso limitado a fuentes de crédito y tecnología, lo que hace que la preocupación de la población por conservar el agua no esté dentro de sus prioridades.

Particularmente en la zona de estudio, se establece que la comunidad tiene como aspecto primordial cubrir las necesidades básicas, dándole 
menor relevancia a mejorar los procesos educacionales de sus integrantes, incrementando sustancialmente los índices de pobreza, y por ende afectando la conservación de los recursos naturales. De allí, la importancia de promover procesos educacionales en la comunidad estudiada; según la UNESCO (2012), una buena educación incluyendo temas ambientales y pedagógicos, estimula a los estudiantes a hacer preguntas, a analizar, a pensar de forma crítica y a tomar decisiones; puesto que la educación de calidad tiene una influencia relevante en el sentido de pertenencia para con el entorno de quien está aprendiendo.

Por otro lado, se precisa que el agua tiene importante relevancia en los procesos de transformación social por todo el potencial productivo que posee, al igual que por sus consecuencias sociales en el funcionamiento de las organizaciones; es por esto, que desempeña un rol significativo dentro del estudio de género y manejo del agua, lo cual ha tomado gran importancia en el desarrollo de las comunidades y los estudios en el tema; así pues, el Programa de las Naciones Unidas para el Desarrollo -PNUD(2006), manifiesta que la situación del agua y el género resulta demasiado familiar, siendo que las mujeres trabajan en la consecución del agua para cubrir las necesidades del hogar, mientras que los hombres toman las decisiones acerca de la gestión y el desarrollo de los recursos hídricos, evidenciándose en un porcentaje mayor en las zonas rurales.

En la comunidad ribereña del río El Encano, las preocupaciones y experiencias de las mujeres, al igual que las de los hombres, deben ser parte integrante de la elaboración, puesta en marcha, control y evaluación de políticas y programas acordes con la gestión del recurso hídrico, ya que en la actualidad es más representativo el papel que el hombre desarrolla; sin embargo, existen ejemplos representativos de mujeres que abanderan procesos ambientales reconocidos tanto nacional como internacionalmente, situación que se ha fortalecido con las experiencias aprendidas.

Por otra parte, considerando los aspectos productivos dentro de la zona de estudio, se hace importante resaltar la contribución económica del turismo; como afirman Muñoz, Fuentes y Fayos-Solá (2012), el turismo puede aportar al desarrollo de la región cuando promueve el uso sostenible de los recursos naturales, físicos y culturales, realzando el capital humano y su productividad socioeconómica, lo que se conoce como ecoturismo. Asimismo, la zona de estudio se convierte en una de las áreas que representan la identidad no solo de la comunidad presente en el área de estudio, sino también de todo el departamento de Nariño.

Según el Banco Mundial (2008), la actividad agrícola es un gran contribuyente al desarrollo de las naciones, al consolidarse como actividad económica, como medio de subsistencia y como proveedor de servicios ambientales; donde la agricultura ha sido el motor del crecimiento de los demás sectores y en general de los países; al respecto de este tema, Gouveia 2011) muestra que los agricultores poseen una característica relevante, como es la de cultivar especies propias de la zona; particularmente, en el área de estudio, esto se observa en algunos aspectos de la producción, ya que la siembra de los productos agrícolas se encuentra ligado a la implementación de las especies nativas, pero no en las actividades acuícolas, donde la diversidad del ecosistema acuático se ha reducido por la intervención del cultivo de truchas, base fundamental de la economía de la región. 
No obstante, con respecto a los aspectos negativos de la agricultura, entidades internacionales como la Organización de las Naciones Unidas para la Agricultura y la Alimentación -FAO- (2006), indican que la agricultura es la causa de deterioro más importante de los cuerpos de agua; ya que los ríos y lagos por estar tan cerca de las comunidades especialmente rurales, se ven afectados por esta actividad; encontrándose cantidades superiores a las permisibles de pesticidas, nutrientes y enriquecimiento de materia orgánica, todos estos componentes acumulados en el agua por los procesos de filtración y percolación del suelo.

En contraste con la afirmación anterior, un enfoque importante para la conservación del agua y del suelo es la participación de los agricultores como elemento clave dentro del funcionamiento de los agroecosistemas, tomando los conocimientos locales adquiridos mediante un proceso de aprendizaje natural; sin embargo, en la actualidad, dado el desarrollo tecnológico y la innovación del conocimiento para el cuidado del recurso hídrico, es necesario que las personas de la región logren conjugar los conocimientos que se obtienen en el hogar, la familia y la comunidad a través de la tradición, con la técnica que se consigue en la academia, de ahí la importancia de la educación para las comunidades de la zona.

Por otra parte, se hace necesario resaltar que pese a que la zona de estudio es de gran importancia ecoturística, carece de infraestructura necesaria para salvaguardar el entorno; como afirma López (2006), para que el desarrollo humano sea sostenible debe contener una serie de factores, entre los cuales se relacionan: ser técnicamente limpio, ecológicamente compatible, económicamente viable y sostenible, y éticamente pertinente. Estos factores, señalados por el autor, no se tienen en cuenta en el desarrollo de esta región considerada turística, puesto que a simple vista no se ve el interés por el cuidado del medio ambiente ni por el buen aspecto físico de la zona en relación con la disposición de residuos sólidos y líquidos.

Otro de los aspectos evaluados en la investigación y pertinente para el desarrollo de la población son los servicios públicos, cuyos resultados mayoritariamente determinan que más del $90 \%$ de las viviendas gozan de servicios primarios fundamentales, como son la disponibilidad del recurso hídrico en un 100\% y la energía en un 99\%; por su parte, el 56\% utilizan gas para cocinar, el cual lo adquieren en los carros repartidores de las empresas existentes en la ciudad de San Juan de Pasto.

Particularmente, en lo concerniente a la potabilización del recurso hídrico objeto de investigación, Saracho et al. (2006) establecen que debe reunir algunos requisitos principales como son: la calidad, que debe observar pautas microbiológicas y fisicoquímicas, que no involucren riesgo para la salud de las personas expuestas, además, debe reunir características relativas a su calidad estética, vinculadas a variables de percepción organoléptica, donde se incluyen los parámetros de calidad con significación estética.

Priorizando en el servicio público de acueducto que provee de agua a las viviendas de esta zona, se obtuvo información de la captación de este recurso para el consumo y uso diario de los habitantes. Los resultados determinan que el $28 \%$ de la población se abastece del acueducto ubicado en la vereda El Socorro, que proviene de la quebrada Torcaza o Salado y el $35 \%$ del que provee a la parte central del corregimiento El Encano, 
tomado de la quebrada Caballo Corral; por lo que se establece que la comunidad no hace uso del agua del río El Encano con fines de consumo humano, sino como el lugar donde se depositan las aguas domésticas. Por lo anterior, tomando en consideración los usos que se hacen del río, Padilla (2012) considera que la comunidad rural desconoce la importancia de mantener los sistemas de agua, conllevando a un nivel de vida bajo, por la presencia de enfermedades infecciosas relacionadas con el consumo de agua.

De allí que el recurso hídrico es fundamental para el desarrollo de la vida del ser humano, de las plantas y de los animales; es por esto, que la generación de conocimientos y el manejo racional del recurso hídrico, contribuye al mejoramiento de la calidad de vida del hombre. Partiendo de esta premisa, se establece que la calidad del agua es uno de los aspectos que más influyen en la condiciones de vida de las poblaciones, principalmente en la población infantil, sobre la cual se logró identificar que, dadas las condiciones del agua, el 54\% de la población ha sufrido enfermedades como gastroenteritis, alergias y problemas dermatológicos, causados por la calidad de dicho recurso.

Según los resultados obtenidos tanto del diagnóstico como de la encuesta realizada a la comunidad sobre los usos del agua, la problemática ambiental y la disposición de aguas residuales y basuras, es claro que los habitantes de la ribera del río El Encano no tienen el conocimiento, ni una clara percepción sobre los usos adecuados y eficientes del agua; esto podría sustentarse en el hecho de que el recurso hídrico es abundante en la región; razón por la que se hace necesario desarrollar estrategias que se encaminen a sensibilizar a las comunidades sobre la educación ambiental y el uso y conservación del recurso hídrico.

De acuerdo a los resultados obtenidos en la zona de estudio, se establece que posee un gran potencial para la agricultura; sin embargo, el bajo nivel educativo de la población, el desinterés por mejorar los procesos en este campo, sumado al conformismo frente al nivel de productividad de sus terrenos, no han permitido mejorar las prácticas en la agricultura. Se constituye, entonces, en factor importante la implementación de estrategias eficaces encaminadas a maximizar la productividad del campo, y a procurar eficientemente la conservación del recurso hídrico. Se requiere, por lo tanto, un trabajo continuo y permanente con las comunidades presentes en el área, que contribuya a mejorar las prácticas de producción que aporten al desarrollo sostenible en la región.

En lo que respecta a la agricultura, principal actividad económica para el sustento de las familias, el agua utilizada para los distintos procesos involucrados en la actividad agrícola se obtiene mayoritariamente en un $61 \%$ del acueducto; el 31\% de los agricultores la toman directamente del río El Encano y el resto, representado en un 8\%, utilizan el agua de pozos cercanos para los procesos agrícolas de sus parcelas, cuyos productos más cultivados en la región y aprovechados para la obtención de recursos económicos son la cebolla en un $40 \%$, la papa en un $33 \%$ y la mora en un $19 \%$, cultivos propios del clima y las características de la zona.

Dentro de este contexto, la Asociación Mundial para el Agua -GWP- y Comité de Consejo Técnico -TAC- (2000) declaran que el uso de este recurso en la agricultura se destina básicamente al riego y la ganadería, siendo el primero de ellos la actividad económica que requiere mayor cantidad de agua, su uso para el riego alcanza casi el $70 \%$ de la demanda 
total. A diferencia de lo anterior, en la zona donde se llevó a cabo la investigación, por los índices de precipitación, no hay necesidad de disponer de agua para este proceso; si fuera así, una gestión adecuada del agua destinada a los procesos del riego, sería necesaria para lograr una eficiencia del aprovechamiento del recurso.

De otra parte, el manejo de los residuos sólidos, tal como lo indican Ayininuola y Muibi (2008), cuando es inadecuado provoca efectos nocivos como contaminación de suelo, aire y agua, deterioro del paisaje natural, menor biodiversidad y riesgos a la salud, entre otros. Particularmente, los residuos generados en la zona de investigación son considerados un problema permanente para el medio ambiente, pues no existe el nivel educativo y cultural requerido, ni la existencia de programas de capacitación sobre su manejo. Estudios desarrollados por Taboada et al. (2011), expresan que la carencia de estudios en zonas rurales no permite dimensionar la problemática ambiental en estas zonas, conduciendo en el mejor de los casos, al desarrollo de sistemas de sanidad rudimentarios o inadecuados.

En lo que respecta al corregimiento El Encano, lugar donde se encuentra el río objeto de estudio, el manejo de los residuos sólidos es desarrollado por la Empresa Metropolitana de Aseo (EMAS) de la ciudad de Pasto, que envía un carro recolector de basura al corregimiento, una vez por semana, lo que ha contribuido en consecuencia a la organización de los residuos sólidos; al respecto, el 70\% de la población encuestada dice depositar las basuras en dicho carro recolector, siendo esta una buena solución para la disposición final de los residuos en la región.

Desde el punto de vista ambiental, los residuos sólidos se constituyen en un problema, debido a que no existen criterios adecuados para seleccionar y ubicar los sitios de disposición final en las zonas rurales; además, es muy frecuente como se evidencia en la zona, que en estos lugares se haga una recolección indiscriminada de todo tipo de residuos, incluyendo los tóxicos y peligrosos que aumentan el riesgo ambiental e incrementan la vulnerabilidad de las personas. Por esto, una buena forma de manejar o de disponer los residuos orgánicos que se generan en las actividades agrícolas o en las actividades internas de las casas, es en abono o en alimento para los animales.

Ligado a la disposición final de los residuos sólidos, están las problemáticas ambientales que se presentan en la región. Las variables evaluadas corresponden, en primera instancia, al recurso hídrico que se constituye en parte de la problemática ambiental. Los encuestados opinaron en un $56 \%$ que el mayor problema ambiental se encuentra en el recurso agua; seguido del recurso suelo con un $40 \%$. Resultados que se corresponden directamente con los problemas planteados sobre depósito de las aguas residuales y manejo de las basuras, además de los residuos generados por la agricultura por la utilización de agroquímicos y fertilizantes.

En este orden de ideas, la Fundación Biodiversidad (2006) argumenta que a medida que se incrementa la población y crece el desarrollo tecnológico, es mayor el deterioro sobre el medio. Así, los problemas ambientales priorizados por los encuestados casi que al mismo nivel son: las aguas residuales con un $46 \%$ y el manejo de las basuras con un $44 \%$. La presencia de esta problemática medioambiental en la zona, indica que el nivel de gestión ambiental en cada uno de los aspectos es deficiente. 


\section{CONCLUSIONES}

Los procesos de gestión del recurso hídrico dentro de la zona de estudio son incipientes, en la medida en que se hace necesaria una intervención activa de las comunidades de la zona, en donde los procesos de sensibilización son fundamentales para lograr optimizar el manejo y el uso del recurso hídrico.

La intervención antrópica en el río El Encano ha sido un factor determinante en la transformación de las características fisicoquímicas y organolépticas de sus aguas, lo cual tiene incidencia importante en la salud de las comunidades y en la alteración de los procesos ecológicos dentro del ecosistema.

El río El Encano, a pesar de ser una de las zonas de mayor importancia para el departamento de Nariño, no cuenta con estrategias eficientes relacionadas con el manejo de las aguas residuales y residuos sólidos, generando consecuencias negativas tanto a la comunidad presente en la zona como al mantenimiento de las condiciones ecosistémicas del área.

\section{POTENCIAL CONFLICTO DE INTERESES}

No hay conflicto de intereses

\section{FUENTES DE FINANCIACIÓN}

El presente artículo es fruto de los resultados de investigación financiada por la Universidad Mariana de la ciudad de Pasto.

\section{REFERENCIAS}

- Araya, E. (2003). Diagnóstico de la calidad del agua en sistemas lóticos utilizando diatomeas y macroinvertebrados bentónicos como bioindicadores, río Maipo. Sociedad Chilena de Ingeniería Hidráulica, XVI Congreso Chileno de Ingeniería Hidráulica, Santiago de Chile, 1314 noviembre.

- Asociación Mundial para el Agua -GWP-y Comité de Consejo Técnico -TAC-. (2000). Manejo integrado de recursos hídricos. Estocolmo: Global Water Partnership.

- Ayininuola, G. y Muibi, M. (2008). An engineering approach to solid waste collection system: Ibadan North as case study. Waste Management, 28(9), 1681-1687. 
- Banco Mundial. (2008). Informe sobre el desarrollo mundial 2008. Agricultura para el desarrollo. Bogotá: Banco Mundial, coeditado con Mundi-Prensa y Mayol Ediciones, S.A.

- Baribbi, A. y Spijkers, P. (2011). Campesinos, tierra y desarrollo rural. Reflexiones desde la experiencia del tercer laboratorio de paz. Bogotá: Asistencia Técnica Internacional del Tercer Laboratorio de Paz, Comisión Europea.

- Departamento Nacional de Estadística -DANE-. (2007). Dirección de censos y demografía. Colombia una nación multicultural. Su diversidad étnica. Bogotá: Autor.

- Fundación Biodiversidad. (2006). Guía de sensibilización de problemas ambientales. Agua: situación y problemática del agua. Madrid: Unión Europea, Fondo Social Europeo.

- Geilfus, F. (2009). 80 herramientas para el desarrollo participativo: Diagnóstico, planificación, monitoreo, evaluación, (Octava ed.). San José, Costa Rica: Instituto Interamericano de Cooperación para la Agricultura -IICA-.

- Gouveia, M. (2011). Criterios de conservación de suelos y aguas en la comunidad rural de Santa Rosa de Ceiba Mocha, Estado Guárico. XIX Congreso Venezolano de la Ciencia del Suelo, Calabozo, 21-25 noviembre.

- Instituto Geográfico Agustín Codazzi -IGAC-. (2014). Geoportal: Visor de mapas. Recuperado de http://www.geoportal.igac.gov.co

- Jaramillo, P. (2006). Pobreza rural en Colombia. Revista Colombiana de Sociología, 27, 47-62.

- López, A. (2006). El desarrollo humano sustentable: bases teóricas y prácticas para la implementación en la Universidad de la Salle. Revista de la Universidad de la Salle, 41, 79-87.

- Muñoz, I., Fuentes, L. y Fayos-Solá, E. (2012). Turismo como instrumento de desarrollo: una visión alternativa desde factores humanos, sociales e institucionales. PASOS, Revista de Turismo y Patrimonio Cultural, 10(5), 437-449.

- Organización de las Naciones Unidas para la Educación, la Ciencia y la Cultura -UNESCO-. (2012). Educación para el Desarrollo Sostenible, libro de consulta. París: Autor.

- Organización para la Agricultura y la Alimentación -FAO. (2006). Introduction to Agricultural Water Pollution. Recuperado de http://www.fao.org/docrep/W2598E/w2598e04.htm

- Padilla, W. (2012). Consumo de agua segura en la Comunidad de Tiopamba del Cantón, Nabón. Tesis de Magíster en Gerencia en Salud para el Desarrollo Local, Universidad Técnica Particular de Loja. Cuenca, Ecuador.

- Programa de las Naciones Unidas para el Desarrollo -PNUD-. (2006). Transversalización del enfoque de género en la gestión del agua. Guía de recursos. Zimbabwe: Autor.

- Saracho, M., Segura, L., Moyano, P., Rodríguez, N. y Carignano, E. (2006). Calidad del agua del Río del Valle, Catamarca, para uso recreativo. Revista de Ciencia y Técnica, Argentina, IX(12), 29-42.

- Taboada, P., Aguilar, Q., Ojeda, S. y Armijo, C. (2011). Estrategia para el manejo de residuos sólidos en una comunidad rural de México. Red de Ingeniería en Saneamiento Ambiental -REDISA-, 175-180. Recuperado http://www.redisa.uji.es/artSim2011/GestionYPoliticaAmbiental/Estrategi a\%20para\%20el\%20manejo\%20de\%20residuos\%20s\%C3\%B3lidos\%2 Oen\%20una\%20comunidad\%20rural\%20de\%20M\%C3\%A9xico.pdf 
- The Ramsar Convention on Wetlands. (2011). A cerca de Ramsar. Recuperado de http://www.ramsar.org/cda/es/ramsar-about-aboutramsar/main/ramsar/1-36\%5E7687_4000_2

- WWF. (2012). Una mirada a la agricultură de Colombia desde su huella hídrica. Reporte Colombia. Cali: WWF Colombia.

1. Magíster en Sistemas Integrados de Gestión. Miembro del Grupo de Investigación Ambiental -GIA-. Universidad Mariana de Pasto. Pasto, Colombia.samaluhe@gmail.com

2. Magíster en Manejo Integrado de Cuencas Hidrográficas. Miembro del Grupo de Investigación Ambiental -GIA-, docente de postgrados. Universidad Mariana de Pasto. Pasto, Colombia. sandritamadro@gmail.com

Para citar este artículo: Luna Hernández, S.M. y Madroñero Palacios, S.M. (2016). Importancia del componente social en el manejo del recurso hídrico, río El Encano, humedal Ramsar La Cocha (Nariño, Colombia). Revista Luna Azul, 42, 200-216. Recuperado de http://200.21.104.25/lunazul/index.php?option=com_content\&view=artic le\&id=135 